\title{
Preliminary Study on Chemical Speciation of Sulphur in Cancerous Tissues
}

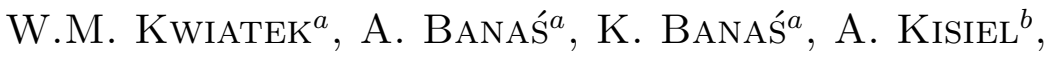 \\ G. Cinque $^{c}$ and G. FAlkenberg ${ }^{d}$ \\ ${ }^{a}$ Institute of Nuclear Physics, Radzikowskiego 152, 31-342 Kraków, Poland \\ ${ }^{b}$ Institute of Physics, Jagiellonian University \\ Reymonta 4, 30-059 Kraków, Poland \\ ${ }^{c}$ Laboratori Nationali di Frascati (LNF) \\ via E. Fermi 40, 00044 Frascati (Rome), Italy \\ ${ }^{d}$ HASYLAB, DESY, Notkestraße 85, 22603 Hamburg, Germany
}

\begin{abstract}
Sulphur is vital for almost all living organisms by participating in a wide variety of metabolic processes. Nevertheless its biochemistry is only partially understood, due to it a few tools such as X-ray absorption near edge structure may be used to determine its chemical speciation in biological system. In this paper we focused on analysis of composition and elemental distribution in tissue structures of biological samples. We examined cancerous and noncancerous prostate tissues. Experiments were carried out on the L-beam line at the HASYLAB, DESY (Hamburg, Germany) and DA $\Phi$ NE-Light Facility DRX1 at the Laboratori Nationali di Frascati (Frascati, Italy). Synchrotron radiation induced X-ray emission measurements reveal the significant differences in concentration of sulphur between cancerous and non-cancerous tissues. X-ray absorption near edge structure spectra show two main types of sulphur that are represented by X-ray peaks at $2476 \mathrm{eV}$ and $2484 \mathrm{eV}$. This suggests that different sulphur components are present in the measured samples.
\end{abstract}

PACS numbers: 87.64.Gb

\section{Introduction}

Sulphur is a required structural mineral found in the proteins of most tissues in the body. It is also necessary for healing and repairing process of these tissues from internal injury and free radical damage. Sulphur takes part in tissue respiration, the process whereby oxygen is used to build new cells, a lack of sulphur 
can lead to replace cells being dysfunctional and causing serious health related problems. This element helps also the immune system to inhibit chemical carcinogens. Sulphur has also a vital relationship with protein, since it can be found in the amino acids methionine, cystine, and cysteine. These amino acids are known as the sulphur-bearing amino acids which are considered as the building blocks of protein. The sulphur-bearing amino acid methionine is absolutely essential to health. The iron-sulphur proteins are also found in a wide variety of organisms and are usually involved in electron transfer processes. These proteins contain one or more iron-sulphur centres, consisting of iron, inorganic sulphur, and/or sulphur atoms from cysteine residues organized in a cluster.

On the other hand sulphur may take part in transfers of free radical centres [1]. The disulphide radical anion appears to be an important terminus of both oxidative and reductive radical transfer [2]. Metal sulfides display usually a higher carcinogenic activity than other derivatives of the same metal. It is probably due to the potential of both the metal cation and the sulfide anion to undergo oxidation and thus activate $\mathrm{O}_{2}^{*}$ or other oxygen species [3]. Understanding the mechanism involved in these processes is already challenging.

Along with the other types of oxidative damage, protein oxidation is believed to be mechanistically involved in aging, the development of ischemia/reperfusion injury, Alzheimer's disease, Parkinson's disease, arteriosclerosis, liver cirrhosis, cancer, etc.

In this paper we used combined synchrotron X-ray micro-fluorescence and X-ray absorption near edge structure (XANES) analyses to illustrate the concentration level and oxidation state for sulphur in prostate tissues. A successful determination of elemental concentration was very important for subsequent XANES measurements. The study of the spatial distribution of sulphur gives a better understanding of the functionality and its chemical properties. The analysis of sulphur surroundings in different kinds of prostate tissues is very important and may give an answer to the questions which proteins are involved in a cancer process.

\section{Materials and methods}

The synchrotron radiation induced X-ray emission (SRIXE) measurements were carried out at the beam line L at DESY in Hamburg, Germany. Synchrotron radiation was produced by $4.5 \mathrm{GeV}$ electron beam with current of $120 \mathrm{~mA}$. The synchrotron radiation from bending magnet was monochromatized by a Si (111) double crystal monochromator set to $17 \mathrm{keV}$.

The samples were positioned at $45^{\circ}$ with respect to the X-ray beam. The beam size was set down to about $15 \mu \mathrm{m}$ in diameter (with the use of the polycapillary). For the calculation of the sulphur concentrations the spectra were recorded for $300 \mathrm{~s}$.

Two-dimensional mappings were performed in order to determine sulphur distribution in different tissue structures. This procedure enabled us to find areas 
with high and homogeneous concentration of $\mathrm{S}$ for later XANES analysis. A detailed description of this method is presented by A. Banaś [4] also published in the same issue of Acta Physica Polonica A.

Sulphur $K$-edge XANES measurements were performed at DA $\Phi$ NE-Light Facility wiggler beamline DRX1 operating with an approximated photon flux of $10^{13}$ photons $/(\mathrm{s} \cdot 0.1 \% \mathrm{BW})$. Due to the high circulating current $(1.8 \mathrm{~A})$, in spite of the low electron beam energy $\left(E_{\mathrm{c}}=308 \mathrm{eV}\right)$, these photon beams will be competitive in respect to other synchrotron radiation (SR) sources in experiments where a strong flux is preferred. The DRX1 beamline allowed us to carry out experiments up to $7 \mathrm{keV}$ of photon energy using a double-crystal fixed exit monochromator [5].

XANES spectra at the $\mathrm{S} K$-edge were recorded in transmission mode. The white synchrotron radiation beam was initially monochromatized set to $2472 \mathrm{eV}$ and then scanned from $2470 \mathrm{eV}$ to $2490 \mathrm{eV}$ around the sulphur $K$-edge in order to acquire the XANES spectrum of this element. The incident X-ray energy was incremented by $0.2 \mathrm{eV}$. Each energy interval was counted for 2 or 5 life time (depending on the intensity of the main edge signal).

In order to determine the chemical nature of $\mathrm{S}$ in the tissues samples, it was crucial to compare the XANES spectra of samples in which the local chemical environments of $\mathrm{S}$ are known. Selected compounds such as $\mathrm{Na}_{2} \mathrm{~S}, \mathrm{Na}_{2} \mathrm{SO}_{3}, \mathrm{Na}_{2} \mathrm{SO}_{4}$, and pure $\mathrm{S}$ were chosen as standards with the appropriate oxidation state of sulphur (S(II), S(IV), $\mathrm{S}(\mathrm{VI})$, and $\mathrm{S}(0)$, respectively). The compounds were chosen because of their physiologically presence in tissues [6].

\section{Results and discussion}

SRIXE measurements reveal the significant differences in concentration of sulphur between cancerous and non-cancerous tissues. In non-cancerous tissue sulphur concentration level is almost by the factor of two larger than in cancerous tissues.

For all experimental XANES spectra a linear pre-edge background was subtracted and then was the edge-jump normalized.

The XANES spectra were analyzed based mainly on shape, position of the pre-edge peak and shift of the absorption edge. The strong differences between the XANES spectra of sulphur compounds can be explained by the local structure around the $\mathrm{S}$ atom and different oxidation states. The near-edge fine structure is unique for each compound and can be compared with the XANES from prostate tissues.

Figure 1 demonstrates the different shapes of XANES spectra depending on structures of analyzed compounds. The absorption edge of most oxidized sulphur is shifted towards a higher energy.

The initial experiments, were performed on $15 \mu \mathrm{m}$ thick samples, but we have found an extremely low signal, due to the low concentration of S. The determination of the speciation of sulphur at this level of concentration was a real challenge. 


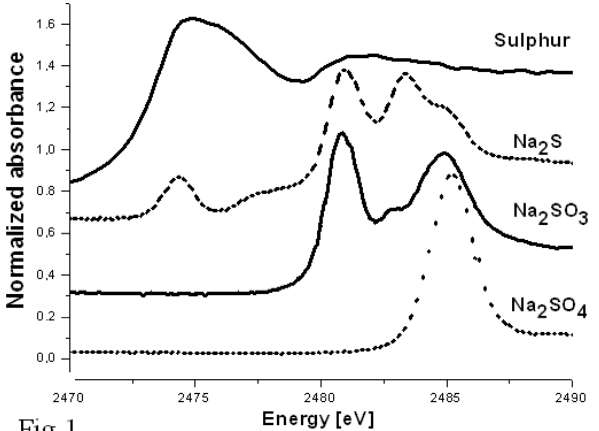

Fig. 1

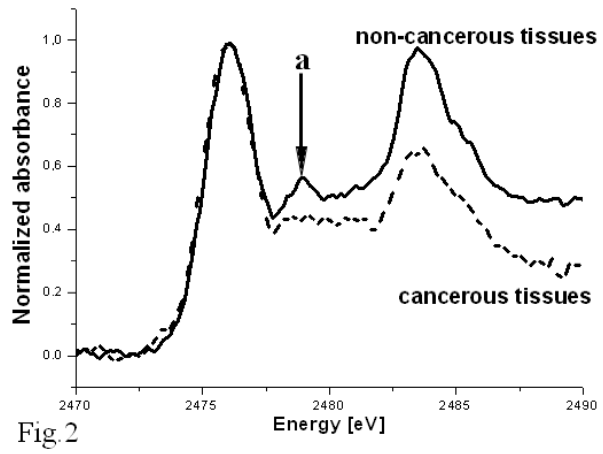

Fig. 2

Fig. 1. Sulphur $K$-edge spectra for reference compounds.

Fig. 2. Sulphur $K$-edge spectra for non-cancerous and cancerous tissues.

The spectra we have recorded from thicker samples $(100 \mu \mathrm{m})$ show a signal-to-noise ratio that looks reasonably well (Fig. 2). XANES spectra for cancerous and non-cancerous tissues show two main types of sulphur with X-ray peaks at $2476 \mathrm{eV}$ and $2484 \mathrm{eV}$. This suggests that in the measured samples different sulphur components are present. The peak at $2484 \mathrm{eV}$ may indicate the presence of sulphur at valence +6 (the sulphate $\mathrm{SO}_{4}^{2-}$ ) or at valence +4 (the sulphite $\mathrm{SO}_{3}^{2-}$ ). The peak at $2476 \mathrm{eV}$ is slightly shifted to a higher energy in comparison with the peak position for pure sulphur.

Additionally the relative intensities between these peaks are unequal for different types of samples. This fact may signify that in cancerous tissues concentration of sulphate or sulphite is at a lower level in reference to non-cancerous tissues. However, the peak $a$ in the spectrum of cancerous tissue presented in Fig. 2 denotes that the sulfonate has been reduced to a new sulphur species. The nature of this species has not been determined yet. A qualitative analysis of the spectra permit us to draw the following conclusions:

- the energy resolution of $0.2 \mathrm{eV}$ provided by the monochromator was sufficient to reveal shifts of the sulphur $K$-edge for prostate tissues, the first peaks at $2476 \mathrm{eV}$ are always located at the same position,

- all the XANES spectra show at least two peaks, located at different energies and owning different intensities. It should be noted that this behaviour seems to be related to $\mathrm{S}$ concentration.

\section{Conclusions}

Following the previous considerations it sometimes happens that morphological differences are not sufficient for distinguishing between cancerous and non-cancerous samples. Hence, an important challenge for physics is to extract important information from tissues using high-resolution, non-destructive, and sensitive techniques. Only the use of synchrotron radiation (SR) provides techniques for 
simultaneous elemental and chemical mapping of tissues. SR offers unique properties not attainable from laboratory sources.

This work represents the systematic application of sulphur chemical analysis with XANES spectroscopy to human tissue samples. The specific aim of this work was to discover the differences in concentration levels and chemical state of sulphur in human prostate tissue samples. We have shown that the XANES analysis performed in transmission mode on elements existing in prostate tissues is possible only if appropriate thickness of the samples is applied. Homogeneity of the elements distribution is also important.

Two more aspects must be fulfilled in order to obtain quantitative information about the structure of investigated compounds: performance of an accurate theoretical analysis of the XANES region and acquirement of a better signal-to-noise ratio.

\section{Acknowledgment}

This work has been supported by HASYLAB, DESY, Hamburg, Germany under project II-02-010, the IHP-Contract HPRI-CT-1999-00040/2001-00140 of the European Commission and by Laboratori Nationali di Frascati, Italy under project TARI $06 \mathrm{Nr}$ 16, contract RII3-CT-2004-506078.

\section{References}

[1] J.R. Landolph, in: Metal Ions in Biological Systems, Eds. H. Sigel, A. Sigel, Vol. 36, Marcel Dekker, New York 1999, p 445.

[2] W.A. Prutz, J. Butler, E.J. Land, A.J. Swallow, Int. J. Radiat. Biol. 55, 539 (1989).

[3] K.S. Kasprzak, Free Radical Biology 8 Medicine 32, 958 (2002).

[4] A. Banaś, K. Banaś, G. Falkenberg, G. Dyduch, W.M. Kwiatek, Acta Phys. Pol. A 109, 323 (2006).

[5] http://www.lnf.infn.it.

[6] B. Gilbert, L. Perfetti, O. Fauchoux, J. Redondo, P.A. Baudat, R. Andres, M. Neumann, S. Steen, D. Gabel, D. Mercanti, M.T. Ciotti, P. Perfetti, G. Margaritondo, G. De Stasio, Phys. Rev. E 62, 1110 (2000). 